\title{
FORWARD-CONVEX CONVERGENCE IN PROBABILITY OF SEQUENCES OF NONNEGATIVE RANDOM VARIABLES
}

\author{
CONSTANTINOS KARDARAS AND GORDAN ŽITKOVIĆ
}

(Communicated by Richard C. Bradley)

\begin{abstract}
For a sequence $\left(f_{n}\right)_{n \in \mathbb{N}}$ of nonnegative random variables, we provide simple necessary and sufficient conditions for convergence in probability of each sequence $\left(h_{n}\right)_{n \in \mathbb{N}}$ with $h_{n} \in \operatorname{conv}\left(\left\{f_{n}, f_{n+1}, \ldots\right\}\right)$ for all $n \in \mathbb{N}$ to the same limit. These conditions correspond to an essentially measure-free version of the notion of uniform integrability.
\end{abstract}

\section{INTRODUCTION}

A growing body of work in applied probability in general, and in the field of mathematical finance in particular, has singled out $\mathbb{L}^{0}$, the Fréchet space of a.s.equivalence classes of random variables topologized by the convergence in probability, as especially important (see, e.g., [3, 8, 12, 15]). The reasons for this are multiple, but if a single commonality is to be found, it would have to be the fact that $\mathbb{L}^{0}$ is essentially measure-free. More precisely, the $\mathbb{L}^{0}$-spaces built over the same measure space with different probabilities will coincide as long as the probabilities are equivalent. The desirability and necessity of the measure-free property in mathematical finance stems from the central tenet of replication (popularized by the work of Black, Scholes, Merton and others) which finds its mathematical expression in the theory of stochastic integration. Since replication amounts to complete removal of risk, the probability measure under which a financial system is modeled should not matter, modulo its negligible sets. On the other hand, given that general stochastic integration does not admit a canonical pathwise definition, we are left with $\mathbb{L}^{0}$ as the only proper setting for the theory. The only other measure-free member of the $\left(\mathbb{L}^{p}\right)_{p \in[0, \infty]}$ family, namely $\mathbb{L}^{\infty}$, turns out to be inadequately small for a large number of modeling tasks.

It is important to note that the interplay between $\mathbb{L}^{0}$, the measure-free property, and stochastic integration reaches further into the history than the relatively recent progress in mathematical finance. The seminal work [14 of Stricker on the semimartingale property under absolutely continuous changes of measures and the

Received by the editors October 17, 2010 and, in revised form, February 3, 2011 and July 16, 011.

2010 Mathematics Subject Classification. Primary 46A16, 46E30, 60A10.

The authors would like to thank Freddy Delbaen and Ted Odell for valuable help, numerous conversations and shared expertise, as well as the anonymous referee for constructive comments and suggestions.

Both authors acknowledge partial support by the National Science Foundation, the first author under award number DMS-0908461, and the second author under award number DMS-0706947. Any opinions, findings and conclusions or recommendations expressed in this material are those of the authors and do not necessarily reflect those of the National Science Foundation. 
celebrated result of Dellacherie and Bichteler (see [1, 2, 7]) on the theory of $\mathbb{L}^{0}$ integrators are but two early examples. Even before that, results related to the measure-free structure of $\mathbb{L}^{0}$, but without relation to stochastic integration, have been published (see, e.g., [4, 13]).

While $\mathbb{L}^{0}$ seems to fit the modeling requirements perfectly, there is a steep price that needs to be paid for its use: a large number of classical functional-analytic tools which were developed for locally convex (and, in particular, Banach) spaces must be renounced. Indeed, $\mathbb{L}^{0}$ fails the local-convexity property in a dramatic fashion: if $(\Omega, \mathcal{F}, \mathbb{P})$ is nonatomic, the topological dual of $\mathbb{L}^{0}$ is trivial (see 9 , Theorem 2.2, p. 18]). Therefore, a new set of tools which do not rely on local convexity (and the related principles such as the Hahn-Banach theorem) are needed to treat even the most basic applied problems. Specifically, convexity has to be "supplied endogenously", leading to various substitutes for indispensable notions such as compactness (see [5, 11, 15]). A central idea behind their introduction is that a passage to a sequence of convex combinations, instead of a more classical passage to a subsequence, yields practically the same analytic benefit, while working much better with the barren structure of $\mathbb{L}^{0}$. The situation is not as streamlined as in the classical case where true subsequences are considered. Indeed, there are examples of sequences $\left(f_{n}\right)_{n \in \mathbb{N}}$ in $\mathbb{L}_{+}^{0}$ (the nonnegative orthant of $\mathbb{L}^{0}$ ) that converge to zero, whereas the set of all possible limits of the convergent sequences $\left(h_{n}\right)_{n \in \mathbb{N}}$ such that $h_{n} \in \operatorname{conv}\left(\left\{f_{n}, f_{n+1}, \ldots\right\}\right)$ is the entire $\mathbb{L}_{+}^{0}$ (see Example 1.2 for details).

It is a goal of the present paper to give necessary and sufficient conditions on a sequence $\left(f_{n}\right)_{n \in \mathbb{N}}$ in $\mathbb{L}_{+}^{0}$ to be forward-convexly convergent, i.e., such that each sequence of its forward-convex combinations (meaning a sequence $\left(h_{n}\right)_{n \in \mathbb{N}}$ with $h_{n} \in \operatorname{conv}\left(\left\{f_{n}, f_{n+1}, \ldots\right\}\right)$ for all $\left.n \in \mathbb{N}\right)$ converges in $\mathbb{L}_{+}^{0}$ to the same limit. Arguably, forward-convex convergence plays as natural a role in $\mathbb{L}^{0}$ as the strong convergence does in $\mathbb{L}^{1}$-spaces. It rules out certain pathological limits and, as will be shown, imposes a measure-free locally convex structure on the sequence. Put simply, it brings the benefits of local convexity to a naturally nonconvex framework.

As far as sufficient conditions for forward-convex convergence are concerned, the reader will quickly think of an example: almost sure convergence of the original sequence will do, for instance. Other than the obvious ones, useful necessary conditions are much harder to come by, and it is therefore surprising that one of our main results has such a simple form. It says, inter alia, that the following two statements are equivalent for sequences in $\mathbb{L}_{+}^{0}$ :

(1) a sequence $\left(f_{n}\right)_{n \in \mathbb{N}}$ is forward-convexly convergent,

$(2)$ there exists a probability measure $\mathbb{Q}$ in the equivalence class that generates the topology of $\mathbb{L}^{0}$ such that $\left(f_{n}\right)_{n \in \mathbb{N}}$ is $\mathbb{L}^{1}(\mathbb{Q})$-convergent.

Effectively, this equivalence identifies forward-convex convergence as an essentially measure-free version of the notion of uniform integrability.

Our main result also shows that failure of forward-convex convergence carries an interesting structure with it. In fact, when an $\mathbb{L}_{+}^{0}$-valued sequence that is $\mathbb{L}^{0}$ convergent to $f \in \mathbb{L}_{+}^{0}$ fails to be forward-convexly convergent, the set $\mathcal{C}$ of all possible limits of its forward-convex combinations is a strict superset of $\{f\}$. A surprising amendment we add to this statement is that $f \leq g$ holds in the almost sure sense for all $g \in \mathcal{C}$; in words, the limit $f$ is always the smallest element in $\mathcal{C}$. 
This extremality property can be viewed as an essentially measure-free no-loss-ofmass condition on the original sequence, giving further support to the interpretation of forward-convex convergence as a variant of uniform integrability.

After this introduction, we give a brief review of the notation and terminology and state our main result in Section 1. The proof of our main result is presented in Section 2 ,

\section{The RESUlT}

1.1. Preliminaries. Let $(\Omega, \mathcal{F}, \overline{\mathbb{P}})$ be a probability space, and let $\Pi$ be the collection of all probabilities on $(\Omega, \mathcal{F})$ that are equivalent to (the representative) $\overline{\mathbb{P}} \in \Pi$. All probabilities in $\Pi$ have the same sets of zero measure, which we shall call $\Pi$-null. We write "П-a.s." to mean $\mathbb{P}$-a.s. with respect to any, and then all, $\mathbb{P} \in \Pi$.

By $\mathbb{L}_{+}$we shall denote the set of all (equivalence classes modulo $\Pi$ of) possibly infinite-valued nonnegative random variables on $(\Omega, \mathcal{F})$. We follow the usual practice of not differentiating between a random variable and the equivalence class it generates in $\mathbb{L}_{+}$. The expectation of $f \in \mathbb{L}_{+}$under $\mathbb{P} \in \Pi$ is denoted by $\mathbb{E}_{\mathbb{P}}[f]$. For fixed $\mathbb{P} \in \Pi$, we define a metric $d_{\mathbb{P}}$ on $\mathbb{L}_{+}$via $d_{\mathbb{P}}(f, g)=\mathbb{E}_{\mathbb{P}}[|\exp (-f)-\exp (-g)|]$ for $f \in \mathbb{L}_{+}$and $g \in \mathbb{L}_{+}$. The topology on $\mathbb{L}_{+}$that is induced by the previous metric does not depend on $\mathbb{P} \in \Pi$; convergence of sequences in this topology is simply (extended) convergence in probability under any $\mathbb{P} \in \Pi$.

A set $\mathcal{C} \subseteq \mathbb{L}_{+}$is convex if $(\alpha f+(1-\alpha) g) \in \mathcal{C}$ whenever $f \in \mathcal{C}, g \in \mathcal{C}$ and $\alpha \in[0,1]$, where the multiplication convention $0 \times \infty=0$ is used. For $\mathcal{A} \subseteq \mathbb{L}_{+}$, $\operatorname{conv}(\mathcal{A})$ denotes the smallest convex set that contains $\mathcal{A} ; \operatorname{conv}(\mathcal{A})$ is just the set of all possible finite convex combinations of elements in $\mathcal{A}$. Further, $\overline{\operatorname{conv}}(\mathcal{A})$ will denote the $\mathbb{L}_{+}$-closure of $\operatorname{conv}(\mathcal{A})$.

The set of all $f \in \mathbb{L}_{+}$such that $\{f=\infty\}$ is $\Pi$-null is denoted by $\mathbb{L}_{+}^{0}$. We endow $\mathbb{L}_{+}^{0}$ with the restriction of the $\mathbb{L}_{+}$-topology; convergence of sequences under this topology is simply convergence in probability under any $\mathbb{P} \in \Pi$. When we write $\mathbb{L}_{+}^{0}-\lim _{n \rightarrow \infty} f_{n}=f$, we tacitly imply that both the sequence $\left(f_{n}\right)_{n \in \mathbb{N}}$ and the limit $f$ are elements of $\mathbb{L}_{+}^{0}$.

1.2. Forward-convex convergence. The following is the central notion of the paper:

Definition 1.1. Let $\left(f_{n}\right)_{n \in \mathbb{N}}$ be a sequence in $\mathbb{L}_{+}$. Any sequence $\left(h_{n}\right)_{n \in \mathbb{N}}$ with the property that $h_{n} \in \operatorname{conv}\left(\left\{f_{n}, f_{n+1}, \ldots\right\}\right)$ for all $n \in \mathbb{N}$ will be called a sequence of forward-convex combinations of $\left(f_{n}\right)_{n \in \mathbb{N}}$.

Since $\mathbb{L}_{+}^{0}$ is not a locally convex space, $\mathbb{L}_{+}^{0}$-convergence of a sequence $\left(f_{n}\right)_{n \in \mathbb{N}}$ does not imply that sequences of forward-convex combinations $\left(f_{n}\right)_{n \in \mathbb{N}} \mathbb{L}_{+}^{0}$-converge to the same limit (or, for that matter, to any limit at all). We give an example of a quite pathological behavior.

Example 1.2. Take $\Omega=(0,1]$ equipped with the Borel $\sigma$-field and Lebesgue measure $\mathbb{P}$, and define the sequence $\left(f_{n}\right)_{n \in \mathbb{N}}$ by

$$
f_{n}=(m-1) 2^{m-1} \mathbb{I}_{\left((k-1) / 2^{m-1}, k / 2^{m-1}\right]},
$$

for $n=2^{m-1}+k-1$ with $m \in \mathbb{N}$ and $1 \leq k \leq 2^{m-1}$. It is straightforward to check that $\mathbb{L}_{+}^{0}-\lim _{n \rightarrow \infty} f_{n}=0$, but as we shall show below, this sequence behaves in a 
strange way: for any $f \in \mathbb{L}_{+}^{0}$, there exists a sequence $\left(h_{n}\right)_{n \in \mathbb{N}}$ of forward-convex combinations of $\left(f_{n}\right)_{n \in \mathbb{N}}$ such that $\mathbb{L}_{+}^{0}-\lim _{n \rightarrow \infty} h_{n}=f$.

We start by noting that it suffices to establish the above claim only for $f \in \mathbb{L}_{+}^{\infty}$ and, consequently, pick $f \in \mathbb{L}_{+}^{\infty}$ with $f \leq M$ for some $M \in \mathbb{R}_{+}$. For each $m \in \mathbb{N}$, let $\mathcal{F}_{m}$ be the $\sigma$-field on $\Omega$ generated by the intervals $\left((k-1) 2^{-m}, k 2^{-m}\right], 1 \leq k \leq 2^{m}$. For $m \in \mathbb{N}$, define $g_{m}:=\mathbb{E}_{\mathbb{P}}\left[f \mid \mathcal{F}_{m}\right]$; by the martingale convergence theorem, $\mathbb{L}_{+}^{0}-\lim _{m \rightarrow \infty} g_{m}=f$. Furthermore,

$$
\begin{aligned}
g_{m} & =\sum_{k=1}^{2^{m}} 2^{m} \mathbb{E}_{\mathbb{P}}\left[f \mathbb{I}_{\left((k-1) / 2^{m}, k / 2^{m}\right]}\right] \mathbb{I}_{\left((k-1) / 2^{m}, k / 2^{m}\right]} \\
& =\sum_{k=1}^{2^{m}} \frac{\mathbb{E}_{\mathbb{P}}\left[f \mathbb{I}_{\left((k-1) / 2^{m}, k / 2^{m}\right]}\right]}{m} f_{2^{m}+k-1 .}
\end{aligned}
$$

Set $\alpha_{m, k}=m^{-1} \mathbb{E}_{\mathbb{P}}\left[f \mathbb{I}_{\left((k-1) / 2^{m}, k / 2^{m}\right]}\right] \in \mathbb{R}_{+}$for $m \in \mathbb{N}$ and $1 \leq k \leq 2^{m}$ so that, for $m \geq M$, we have

$$
\sum_{k=1}^{2^{m}} \alpha_{m, k}=\frac{\mathbb{E}_{\mathbb{P}}[f]}{m} \leq \frac{M}{m} \leq 1
$$

Define the sequence $\left(h_{n}\right)_{n \in \mathbb{N}}$ as follows: for $m \in \mathbb{N}$ with $m<M$, simply set $h_{2^{m-1}+k-1}=f_{2^{m-1}+k-1}$ for all $1 \leq k \leq 2^{m-1}$, while for $m \in \mathbb{N}$ with $m \geq M$, set

$$
h_{2^{m-1}+k-1}=\left(1-\sum_{\ell=1}^{2^{m}} \alpha_{m, \ell}\right) f_{2^{m}}+\sum_{k=1}^{2^{m}} \alpha_{m, \ell} f_{2^{m}+\ell-1}=\left(1-\frac{\mathbb{E}_{\mathbb{P}}[f]}{m}\right) f_{2^{m}}+g_{m}
$$

for all $1 \leq k \leq 2^{m-1}$. Then, $\left(h_{n}\right)_{n \in \mathbb{N}}$ is a sequence of forward-convex combinations of $\left(f_{n}\right)_{n \in \mathbb{N}}$, and $\mathbb{L}_{+}^{0}-\lim _{n \rightarrow \infty} h_{n}=f$.

In the above example, note that the limit of $\left(f_{n}\right)_{n \in \mathbb{N}}$ is clearly minimal (in the П-a.s. sense) in the set of all possible limits of sequences of forward-convex combinations of $\left(f_{n}\right)_{n \in \mathbb{N}}$. As Theorem 1.3 will reveal, this did not happen by chance.

1.3. The main result. Having introduced all the ingredients, we are ready to state our main equivalence result.

Theorem 1.3. Let $\left(f_{n}\right)_{n \in \mathbb{N}}$ be a sequence in $\mathbb{L}_{+}^{0}$. Assume that $(\mathrm{CONV})$

$$
\mathbb{L}_{+}^{0}{ }_{-} \lim _{n \rightarrow \infty} f_{n}=f
$$

holds for some $f \in \mathbb{L}_{+}^{0}$. Then, the following three statements are equivalent:

(1) Every sequence of forward-convex combinations of $\left(f_{n}\right)_{n \in \mathbb{N}} \mathbb{L}_{+}^{0}$-converges to $f$.

(2) Whenever a sequence of forward-convex combinations of $\left(f_{n}\right)_{n \in \mathbb{N}}$ is $\mathbb{L}_{+}$convergent, its $\mathbb{L}_{+}$-limit is $f$.

(3) There exists $\mathbb{Q} \in \Pi$ such that $\sup _{n \in \mathbb{N}} \mathbb{E}_{\mathbb{Q}}\left[f_{n}\right]<\infty$ and $\lim _{n \rightarrow \infty} \mathbb{E}_{\mathbb{Q}}\left[\mid f_{n}-\right.$ $f \mid]=0$.

- With (CONV holding, and under any of the above equivalent conditions, we have

$$
\overline{\operatorname{conv}}\left(\left\{f_{1}, f_{2}, \ldots\right\}\right)=\left\{\sum_{n \in \mathbb{N}} \alpha_{n} f_{n}+\left(1-\sum_{n \in \mathbb{N}} \alpha_{n}\right) f \mid\left(\alpha_{n}\right)_{n \in \mathbb{N}} \in \triangle^{\mathbb{N}}\right\},
$$


where $\triangle^{\mathbb{N}}$ is the infinite-dimensional simplex:

$\triangle^{\mathbb{N}}:=\left\{\alpha=\left(\alpha_{n}\right)_{n \in \mathbb{N}} \mid \alpha_{n} \in \mathbb{R}_{+}\right.$for all $n \in \mathbb{N}$, and $\left.\sum_{n \in \mathbb{N}} \alpha_{n} \leq 1\right\}$.

Furthermore, if a measure $\mathbb{Q} \in \Pi$ satisfies condition (3) above, then the set $\overline{\operatorname{conv}}\left(\left\{f_{1}, f_{2}, \ldots\right\}\right)$ is $\mathbb{L}_{+}^{1}(\mathbb{Q})$-compact and the $\mathbb{L}_{+}^{0}$-topology and the $\mathbb{L}_{+}^{1}(\mathbb{Q})$-topology coincide on $\overline{\operatorname{conv}}\left(\left\{f_{1}, f_{2}, \ldots\right\}\right)$. In particular, under the $\mathbb{L}_{+}^{0}$-topology, $\overline{\operatorname{conv}}\left(\left\{f_{1}, f_{2}, \ldots\right\}\right)$ is locally convex and compact.

- With (CONV holding, if any of the equivalent conditions above fail, the set $\mathcal{C} \subseteq \mathbb{L}_{+}$of all possible $\mathbb{L}_{+}$-limits of forward-convex combinations of $\left(f_{n}\right)_{n \in \mathbb{N}}$ is such that $\{f\} \varsubsetneqq \mathcal{C}$, and $f$ is minimal in $\mathcal{C}$ in the sense that $f \leq g$ holds $\Pi$-a.s. for all $g \in \mathcal{C}$.

In the special case $f=0$, the equivalences of the above three statements and the properties discussed after them hold even without assumption (CONV).

Implications $(1) \Rightarrow(2)$ and $(3) \Rightarrow(1)$ are straightforward, and (CONV) is not required. Indeed, $(1) \Rightarrow(2)$ is completely trivial. Also, the implication $(3) \Rightarrow(1)$ is immediate since

$$
\limsup _{n \rightarrow \infty} \mathbb{E}_{\mathbb{Q}}\left[\left|h_{n}-f\right|\right] \leq \limsup _{n \rightarrow \infty}\left(\sup _{\mathbb{N} \ni k \geq n} \mathbb{E}_{\mathbb{Q}}\left[\left|f_{k}-f\right|\right]\right)=0
$$

holds for any sequence $\left(h_{n}\right)_{n \in \mathbb{N}}$ of forward-convex combinations of $\left(f_{n}\right)_{n \in \mathbb{N}}$. The proof of the implication $(2) \Rightarrow(3)$ is significantly harder and will be discussed in Section 2

Remark 1.4. Consider an $\mathbb{L}_{+}^{0}$-convergent sequence $\left(f_{n}\right)_{n \in \mathbb{N}}$, and let $f$ be its limit $f:=\mathbb{L}_{+}^{0}-\lim _{n \rightarrow \infty} f_{n}$. From a qualitative viewpoint, Theorem 1.3 aids our understanding of the cases where a sequence $\left(h_{n}\right)_{n \in \mathbb{N}}$ of forward-convex combinations of $\left(f_{n}\right)_{n \in \mathbb{N}} \mathbb{L}_{+}^{0}$-converges to a limit other than $f$. Indeed, in those cases $f$ is "suboptimal" in a very strong sense: all other possible limits of sequences of forward-convex combinations of $\left(f_{n}\right)_{n \in \mathbb{N}}$ dominate it in the $\Pi$-a.s. pointwise sense.

Remark 1.5. In the special case $f=0$, (CONV is not needed in Theorem 1.3 . However, when $f \neq 0$, CONV is crucial for $(2) \Rightarrow(1)$ of Theorem 1.3 to hold. We present an example to illustrate this fact. Assume that $(\Omega, \mathcal{F}, \mathbb{P})$ is rich enough to accommodate a sequence $\left(f_{n}\right)_{n \in \mathbb{N}}$ of random variables that are independent under $\mathbb{P}$ and have identical distributions given by $\mathbb{P}\left[f_{n}=0\right]=\mathbb{P}\left[f_{n}=2\right]=1 / 2$. By Kolmogorov's zero-one law, it follows that any possible $\mathbb{L}_{+}$-limit of sequences of convex combinations of $\left(f_{n}\right)_{n \in \mathbb{N}}$ has to be constant. Now, $\left(f_{n}\right)_{n \in \mathbb{N}}$ is uniformly integrable (in fact, uniformly bounded) under $\mathbb{P}$, which means that the set $\mathcal{C}$ of all possible $\mathbb{L}_{+}$-limits of sequences of convex combinations of $\left(f_{n}\right)_{n \in \mathbb{N}}$ is $\mathcal{C}=\{1\}$. With $f=1$ we have (2) of Theorem 1.3 holding. However, both (1) and (3) fail.

Remark 1.6. The reader will note that we state our main result, Theorem 1.3 , under the assumption that all $f_{n}$ are nonnegative. While this assumption seems to be crucial for our method of proof to apply, we were unable to construct a counterexample to the appropriately modified version of the theorem for a general sequence $\left(f_{n}\right)_{n \in \mathbb{N}} \subseteq \mathbb{L}^{0}$.

Remark 1.7. Forward-convexly convergent sequences appear quite naturally in applications. Indeed, it has been shown in [6, Proposition A1.1] that every sequence 
in $\mathbb{L}_{+}^{0}$ whose convex hull is bounded in probability admits an a.s.-convergent (and therefore forward-convexly convergent) sequence of forward-convex combinations. This statement can also be deduced from Komlós' lemma (see [1]).

\section{Proof of Theorem 1.3}

2.1. Preparatory remarks. We start by mentioning a result [6, Lemma A1.1] which will be used in a few places throughout the proof of Theorem 1.3. Recall that a set $\mathcal{B} \subseteq \mathbb{L}_{+}^{0}$ is called $\mathbb{L}_{+}^{0}$-bounded if $\downarrow \lim _{\ell \rightarrow \infty} \sup _{f \in \mathcal{B}} \mathbb{P}[f>\ell]=0$ holds for some (and then for all) $\mathbb{P} \in \Pi$. If $\mathcal{B} \subseteq \mathbb{L}_{+}^{0}$ is $\mathbb{L}_{+}^{0}$-bounded, its $\mathbb{L}_{+}$-closure is a subset of $\mathbb{L}_{+}^{0}$ and coincides with its $\mathbb{L}_{+}^{0}$-closure.

Lemma 2.1. Let $\left(g_{n}\right)_{n \in \mathbb{N}}$ be an $\mathbb{L}_{+}^{0}$-valued sequence. Then, there exists $h \in \mathbb{L}_{+}$ and a sequence $\left(h_{n}\right)_{n \in \mathbb{N}}$ of forward-convex combinations of $\left(g_{n}\right)_{n \in \mathbb{N}}$ such that $\mathbb{L}_{+}$$\lim _{n \rightarrow \infty} h_{n}=h$. If, furthermore, conv $\left\{g_{n} \mid n \in \mathbb{N}\right\}$ is $\mathbb{L}_{+}^{0}$-bounded, then $h \in \mathbb{L}_{+}^{0}$.

We introduce some notation that will be used throughout the proof: for $n \in \mathbb{N}$, set $\mathcal{C}_{n}:=\overline{\operatorname{conv}}\left(\left\{f_{n}, f_{n+1}, \ldots\right\}\right) \subseteq \mathbb{L}_{+}$so that $\mathcal{C}=\bigcap_{n \in \mathbb{N}} \mathcal{C}_{n}$. Also, let $\mathcal{S}_{n} \subseteq \mathbb{L}_{+}$be the solid hull of $\mathcal{C}_{n}: g \in \mathcal{S}_{n}$ if and only if $0 \leq g \leq h$ for some $h \in \mathcal{C}_{n}$. It is clear that $\mathcal{S}_{n}$ is convex and solid and that $\mathcal{C}_{n} \subseteq \mathcal{S}_{n}$. Furthermore, set $\mathcal{C}:=\bigcap_{n \in \mathbb{N}} \mathcal{C}_{n} \subseteq$ $\mathbb{L}_{+}$. It is clear that $\mathcal{C}$ is the set of all possible $\mathbb{L}_{+}$-limits of sequences of forwardconvex combinations of $\left(f_{n}\right)_{n \in \mathbb{N}}$. In particular, condition (2) of Theorem 1.3 can be succinctly written as $\mathcal{C}=\{f\}$.

We shall split the proof into several steps, indicating each time what is being proved or discussed. Until the end of subsection 2.3, condition (CONV) is not assumed.

2.2. $\mathcal{C} \subseteq \mathbb{L}_{+}^{0}$ implies that $\overline{\operatorname{conv}}\left(\left\{f_{1}, f_{2}, \ldots\right\}\right)$ is $\mathbb{L}_{+}^{0}$-bounded. We start by showing that $\mathcal{S}_{n}$ is $\mathbb{L}_{+}$-closed, for $n \in \mathbb{N}$. For that, we pick an $\mathcal{S}_{n}$-valued sequence $\left(g_{k}\right)_{k \in \mathbb{N}}$ that converges $\mathbb{P}$-a.s. to $g \in \mathbb{L}_{+}$. Let $\left(h_{k}\right)_{k \in \mathbb{N}}$ be a $\mathcal{C}_{n}$-valued sequence with $g_{k} \leq h_{k}$ for all $k \in \mathbb{N}$. By Lemma 2.1, we can extract a sequence $\left(\widetilde{h}_{k}\right)_{k \in \mathbb{N}}$ of forward-convex combinations of $\left(h_{k}\right)_{k \in \mathbb{N}}$ such that $h:=\lim _{k \rightarrow \infty} \widetilde{h}_{k} \in \mathbb{L}_{+}$I-a.s. exists. Of course, $h \in \mathcal{C}_{n}$, and it is straightforward that $g \leq h$. We conclude that $g \in \mathcal{S}_{n}$; i.e., $\mathcal{S}_{n}$ is $\mathbb{L}_{+}$-closed.

Let $\mathcal{S}=\bigcap_{n \in \mathbb{N}} \mathcal{S}_{n}$; then, $\mathcal{C} \subseteq \mathcal{S}$ and $\mathcal{S}$ is $\mathbb{L}_{+}$-closed, convex and solid. We claim that $\mathcal{S}$ actually is the solid hull of $\mathcal{C}$; to show this, we only need to establish that for any $g \in \mathcal{S}$ there exists $h \in \mathcal{C}$ with $g \leq h$. For all $n \in \mathbb{N}$, since $g \in \mathcal{S} \subseteq \mathcal{S}_{n}$, there exists $h_{n} \in \mathcal{C}_{n}$ with $g \leq h_{n}$. By another application of Lemma 2.1, we can extract a sequence $\left(\widetilde{h}_{n}\right)_{n \in \mathbb{N}}$ of forward-convex combinations of $\left(h_{n}\right)_{n \in \mathbb{N}}$ such that $h:=\mathbb{L}_{+-} \lim _{k \rightarrow \infty} \widetilde{h}_{k}$ exists. Then, $h \in \mathcal{C}$ and $g \leq h$.

Each $\mathcal{S}_{n}$ is $\mathbb{L}_{+}$-closed, convex and solid; therefore, a straightforward generalization of [3. Lemma 2.3] gives, for each $n \in \mathbb{N}$, the existence of a partition $\Omega=$ $\Phi_{n} \cup\left(\Omega \backslash \Phi_{n}\right)$, where $\Phi_{n} \in \mathcal{F},\left\{f \mathbb{I}_{\Phi_{n}} \mid f \in \mathcal{S}_{n}\right\}$ is $\mathbb{L}_{+}^{0}$-bounded, while $h \mathbb{I}_{\Omega \backslash \Phi_{n}} \in \mathcal{S}_{n}$ for all $h \in \mathbb{L}_{+}$. Clearly, $\mathcal{C}_{n} \supseteq \mathcal{C}_{n+1}$ implies $\Phi_{n} \subseteq \Phi_{n+1}$, for all $n \in \mathbb{N}$. However, since $f_{n} \in \mathbb{L}_{+}^{0}$, i.e., $\left\{f_{n}=\infty\right\}$ is $\Pi$-null for all $n \in \mathbb{N}$, it follows that $\Phi_{n+1}=\Phi_{n}$ for all $n \in \mathbb{N}$. In other words, $\Phi_{n}=\Phi_{1}$ for all $n \in \mathbb{N}$. Then, $h \mathbb{I}_{\Omega \backslash \Phi_{1}} \in \mathcal{S}$ for all $h \in \mathbb{L}_{+}$. Since $\mathcal{C} \subseteq \mathbb{L}_{+}^{0}$, and, therefore, $\mathcal{S} \subseteq \mathbb{L}_{+}^{0}$ as well, it follows that $\Omega \backslash \Phi_{1}$ is $\Pi$-null. Therefore, $\mathcal{S}_{1}$ is $\mathbb{L}_{+}^{0}$-bounded, which completes this part of the proof. Observe that all $\mathcal{S}_{n}, n \in \mathbb{N}$, are convex, solid, $\mathbb{L}_{+}^{0}$-bounded, and $\mathbb{L}_{+}^{0}$-closed; we shall use this later. 
2.3. Equivalence of (1), (2) and (3) in Theorem 1.3 when $f=0$. As already discussed, the proofs of $(1) \Rightarrow(2)$ and $(3) \Rightarrow(1)$ are immediate, and (CONV) is not used. Here, we prove $(2) \Rightarrow(3)$ when $f=0$ without assuming (CONV).

Since $\mathcal{S}_{1}$ is convex, $\mathbb{L}_{+}^{0}$-bounded and $\mathbb{L}_{+}^{0}$-closed, there exists $\mathbb{P} \in \Pi$ such that $\sup _{h \in \mathcal{S}_{1}} \mathbb{E}_{\mathbb{P}}[h]<\infty$. Although this result is somewhat folklore, we provide here a quick argument for its validity. Fixing a baseline probability $\overline{\mathbb{P}} \in \Pi,[10$, Theorem 1.1(4)] implies that there exists $\widehat{h} \in \mathcal{S}_{1}$ such that $\mathbb{E}_{\overline{\mathbb{P}}}[h /(1+\widehat{h})] \leq 1$ holds for all $h \in \mathcal{S}_{1}$. Define $\mathbb{P}$ via the recipe $\mathrm{d} \mathbb{P} / \mathrm{d} \overline{\mathbb{P}}=c /(1+\widehat{h})$, where $c=1 / \mathbb{E}_{\overline{\mathbb{P}}}[1 /(1+\widehat{h})]$. Then, $\mathbb{P} \in \Pi$ and

$$
\sup _{h \in \mathcal{S}_{1}} \mathbb{E}_{\mathbb{P}}[h]=c \sup _{h \in \mathcal{S}_{1}} \mathbb{E}_{\overline{\mathbb{P}}}\left[\frac{h}{1+\widehat{h}}\right] \leq c<\infty .
$$

In particular, we have $\sup _{n \in \mathbb{N}} \mathbb{E}_{\mathbb{P}}\left[f_{n}\right]<\infty$. Given the existence of such a $\mathbb{P} \in \Pi$, the following result will be useful in order to extract a probability $\mathbb{Q} \in \Pi$ that satisfies condition (3) of Theorem 1.3

Lemma 2.2. Fix $\mathbb{P} \in \Pi$ with $\sup _{n \in \mathbb{N}} \mathbb{E}_{\mathbb{P}}\left[f_{n}\right]<\infty$. Then, the following statements are equivalent:

(1) For some $\mathbb{Q} \in \Pi$, $\sup _{n \in \mathbb{N}} \mathbb{E}_{\mathbb{Q}}\left[f_{n}\right]<\infty$ and $\lim _{n \rightarrow \infty} \mathbb{E}_{\mathbb{Q}}\left[f_{n}\right]=0$.

(2) For any $\epsilon>0$, there exists $A_{\epsilon} \in \mathcal{F}$ such that $\lim _{n \rightarrow \infty} \mathbb{E}_{\mathbb{P}}\left[f_{n} \mathbb{I}_{A_{\epsilon}}\right]=0$ and $\mathbb{P}\left[\Omega \backslash A_{\epsilon}\right] \leq \epsilon$.

Proof. First assume (1) in the statement of Lemma 2.2. Define $Z:=\mathrm{d} \mathbb{Q} / \mathrm{dP}$; then, $\mathbb{P}[Z>0]=1$. For fixed $\epsilon>0$, let $\delta=\delta(\epsilon)>0$ be such that, with $A_{\epsilon}:=\{Z>\delta\} \in$ $\mathcal{F}, \mathbb{P}\left[\Omega \backslash A_{\epsilon}\right] \leq \epsilon$ holds. Then,

$$
\limsup _{n \rightarrow \infty} \mathbb{E}_{\mathbb{P}}\left[f_{n} \mathbb{I}_{A_{\epsilon}}\right]=\limsup _{n \rightarrow \infty} \mathbb{E}_{\mathbb{Q}}\left[(1 / Z) f_{n} \mathbb{I}_{\{Z>\delta\}}\right] \leq(1 / \delta) \limsup _{n \rightarrow \infty} \mathbb{E}_{\mathbb{Q}}\left[f_{n}\right]=0 .
$$

Now, assume (2) in the statement of Lemma 2.2. For each $k \in \mathbb{N}$, let $B_{k} \in \mathcal{F}$ be such that $\mathbb{P}\left[\Omega \backslash B_{k}\right] \leq 1 / k$ and $\lim _{n \rightarrow \infty} \mathbb{E}_{\mathbb{P}}\left[f_{n} \mathbb{I}_{B_{k}}\right]=0$. By replacing $B_{k}$ with $\bigcup_{m=1}^{k} B_{m}$ for each $k \in \mathbb{N}$ consecutively, we may assume without loss of generality that $\left(B_{k}\right)_{k \in \mathbb{N}}$ is a nondecreasing sequence of sets in $\mathcal{F}$ with $\lim _{k \rightarrow \infty} \mathbb{P}\left[B_{k}\right]=1$, as well as that $\lim _{n \rightarrow \infty} \mathbb{E}_{\mathbb{P}}\left[f_{n} \mathbb{I}_{B_{k}}\right]=0$ holds for each fixed $k \in \mathbb{N}$. Define $B_{0}=\emptyset$, $n_{0}=0$, and a strictly increasing $\mathbb{N}$-valued sequence $\left(n_{k}\right)_{k \in \mathbb{N}}$ with the following property: for all $k \in \mathbb{N}, \mathbb{E}_{\mathbb{P}}\left[f_{n} \mathbb{I}_{B_{k}}\right] \leq 1 / k$ holds for all $n \geq n_{k-1}$. (Observe that this is trivially valid for $k=1$.) Then, define a sequence $\left(E_{n}\right)_{n \in \mathbb{N}}$ of sets in $\mathcal{F}$ by setting $E_{n}=B_{k}$ whenever $n_{k-1} \leq n<n_{k}$. It is clear that $\left(E_{n}\right)_{n \in \mathbb{N}}$ is a nondecreasing sequence, that $\lim _{n \rightarrow \infty} \mathbb{P}\left[E_{n}\right]=1$, and that $\lim _{n \rightarrow \infty} \mathbb{E}_{\mathbb{P}}\left[f_{n} \mathbb{I}_{E_{n}}\right]=0$. With $E_{0}:=\emptyset$, define $Z:=c \sum_{n \in \mathbb{N}} 2^{-n} \mathbb{I}_{E_{n} \backslash E_{n-1}}$, where $c>0$ is a normalizing constant in order to ensure that $\mathbb{E}_{\mathbb{P}}[Z]=1$. Define $\mathbb{Q} \in \Pi$ via $\mathrm{d} \mathbb{Q} / \mathrm{d} \mathbb{P}=Z$ and let $K:=\sup _{n \in \mathbb{N}} \mathbb{E}_{\mathbb{P}}\left[f_{n}\right]<\infty$ so that $\sup _{n \in \mathbb{N}} \mathbb{E}_{\mathbb{Q}}\left[f_{n}\right] \leq c \sup _{n \in \mathbb{N}} \mathbb{E}_{\mathbb{P}}\left[f_{n}\right]=c K<\infty$. Furthermore,

$$
\begin{aligned}
\mathbb{E}_{\mathbb{Q}}\left[f_{n}\right] & =\mathbb{E}_{\mathbb{Q}}\left[f_{n} \mathbb{I}_{E_{n}}\right]+\mathbb{E}_{\mathbb{Q}}\left[f_{n} \mathbb{I}_{\Omega \backslash E_{n}}\right] \leq c \mathbb{E}_{\mathbb{P}}\left[f_{n} \mathbb{I}_{E_{n}}\right]+c 2^{-n} \mathbb{E}_{\mathbb{P}}\left[f_{n} \mathbb{I}_{\Omega \backslash E_{n}}\right] \\
& \leq c \mathbb{E}_{\mathbb{P}}\left[f_{n} \mathbb{I}_{E_{n}}\right]+c K 2^{-n} .
\end{aligned}
$$

Since $\lim _{n \rightarrow \infty} \mathbb{E}_{\mathbb{P}}\left[f_{n} \mathbb{I}_{E_{n}}\right]=0$, we obtain $\lim _{n \rightarrow \infty} \mathbb{E}_{\mathbb{Q}}\left[f_{n}\right]=0$, which completes the argument. 
We continue with the proof of the implication $(2) \Rightarrow(3)$, fixing $\mathbb{P} \in \Pi$ with $\sup _{n \in \mathbb{N}} \mathbb{E}_{\mathbb{P}}\left[f_{n}\right]<\infty$ until the end of subsection 2.3 .

For any $\mathcal{A} \subseteq \mathbb{L}_{+}^{0}$, define its polar $\mathcal{A}^{\circ}:=\left\{g \in \mathbb{L}_{+}^{0} \mid \mathbb{E}_{\mathbb{P}}[g h] \leq 1\right.$ for all $\left.h \in \mathcal{A}\right\}$. It is straightforward that $\left(\bigcup_{n \in \mathbb{N}} \mathcal{A}_{n}\right)^{\circ}=\bigcap_{n \in \mathbb{N}} \mathcal{A}_{n}^{\circ}$, for all collections $\left\{\mathcal{A}_{n} \mid n \in \mathbb{N}\right\}$ of subsets of $\mathbb{L}_{+}^{0}$. Also, consider the bipolar $\mathcal{A}^{\circ \circ}:=\left(\mathcal{A}^{\circ}\right)^{\circ}$ of $\mathcal{A}$; Theorem 1.3 of [3] states that if a set is convex and solid, $\mathcal{A}^{\circ \circ}$ coincides with the $\mathbb{L}_{+}^{0}$-closure of $\mathcal{A}$.

For each $n \in \mathbb{N}, \mathcal{S}_{n} \subseteq \mathbb{L}_{+}^{0}$ is convex, solid and $\mathbb{L}_{+}^{0}$-closed; therefore, $\mathcal{S}_{n}^{\circ \circ}=\mathcal{S}_{n}$. Since $\mathcal{S}=\bigcap_{n \in \mathbb{N}} \mathcal{S}_{n}$ is the solid hull of $\mathcal{C}=\{0\}$, i.e., $\mathcal{S}=\{0\}$, we have

$$
\left(\bigcup_{n \in \mathbb{N}} \mathcal{S}_{n}^{\circ}\right)^{\circ \circ}=\left(\bigcap_{n \in \mathbb{N}} \mathcal{S}_{n}^{\circ \circ}\right)^{\circ}=\left(\bigcap_{n \in \mathbb{N}} \mathcal{S}_{n}\right)^{\circ}=\{0\}^{\circ}=\mathbb{L}_{+}^{0} .
$$

Since $\bigcup_{n \in \mathbb{N}} \mathcal{S}_{n}^{\circ}$ is convex and solid, the above means that the $\mathbb{L}_{+}^{0}$-closure of $\bigcup_{n \in \mathbb{N}} \mathcal{S}_{n}^{\circ}$ is $\mathbb{L}_{+}^{0}$.

Fix $\epsilon>0$. Define an $\mathbb{N}$-valued and strictly increasing sequence $\left(n_{k}\right)_{k \in \mathbb{N}}$ with the following property: for all $k \in \mathbb{N}$ there exists $g_{k} \in \mathcal{S}_{n_{k}}^{\circ}$ such that $\mathbb{P}\left[\left|g_{k}-2 k\right| \leq k\right] \leq$ $\epsilon 2^{-(k+1)}$. (This can be done in view of the fact that the $\mathbb{L}_{+}^{0}$-closure of $\bigcup_{n \in \mathbb{N}} \mathcal{S}_{n}^{\circ}$ is $\mathbb{L}_{+}^{0}$.) In particular, $\mathbb{P}\left[g_{k} \leq k\right] \leq \epsilon 2^{-(k+1)}$ and $\mathbb{E}_{\mathbb{P}}\left[g_{k} f_{n}\right] \leq 1$ hold for all $k \in \mathbb{N}$ and $n \geq n_{k}$. Define $A_{\epsilon}:=\bigcap_{k \in \mathbb{N}}\left\{g_{k}>k\right\}$; then, $\mathbb{P}\left[\Omega \backslash A_{\epsilon}\right] \leq \epsilon$. Furthermore, for all $k \in \mathbb{N}$ and $n \geq n_{k}$,

$$
\mathbb{E}_{\mathbb{P}}\left[f_{n} \mathbb{I}_{A_{\epsilon}}\right] \leq \mathbb{E}_{\mathbb{P}}\left[f_{n} \mathbb{I}_{\left\{g_{k}>k\right\}}\right] \leq \mathbb{E}_{\mathbb{P}}\left[\left(g_{k} / k\right) f_{n} \mathbb{I}_{\left\{g_{k}>k\right\}}\right] \leq(1 / k) \mathbb{E}_{\mathbb{P}}\left[g_{k} f_{n}\right] \leq 1 / k .
$$

Then, $\lim _{n \rightarrow \infty} \mathbb{E}_{\mathbb{P}}\left[f_{n} \mathbb{I}_{A_{\epsilon}}\right]=0$. Invoking Lemma 2.2, we obtain the existence of $\mathbb{Q} \in \Pi$ such that $\sup _{n \in \mathbb{N}} \mathbb{E}_{\mathbb{Q}}\left[f_{n}\right]<\infty$ and $\lim _{n \rightarrow \infty} \mathbb{E}_{\mathbb{Q}}\left[f_{n}\right]=0$.

2.4. A domination result. The next simple result will be important for establishing the validity of Theorem 1.3 .

Proposition 2.3. Let $\left(f_{n}\right)_{n \in \mathbb{N}}$ satisfy (CONV). Furthermore, let $\left(g_{n}\right)_{n \in \mathbb{N}}$ be a sequence of forward-convex combinations of $\left(f_{n}\right)_{n \in \mathbb{N}}$ such that $\mathbb{L}_{+}-\lim _{n \rightarrow \infty} g_{n}=g$. Then, П-a.s., $f \leq g$.

Proof. By way of a contradiction, assume that $\overline{\mathbb{P}}[f>g]>0$, where $\overline{\mathbb{P}} \in \Pi$, and consider the probability $\mathbb{P}$ which is $\overline{\mathbb{P}}$ conditioned on the event $\{f>g\}$. Note that $\mathbb{P}[f>g]=1$ and that $\lim _{n \rightarrow \infty} f_{n}=f$ and $\lim _{n \rightarrow \infty} g_{n}=g$ still hold under the measure $\mathbb{P}$. Let $U:[0, \infty] \mapsto[0,1]$ be the strictly increasing and concave function defined via $U(x)=1-\exp (-x)$ for $x \in[0, \infty]$. The dominated convergence theorem implies that $\lim _{n \rightarrow \infty} \mathbb{E}_{\mathbb{P}}\left[U\left(f_{n}\right)\right]=\mathbb{E}_{\mathbb{P}}[U(f)]$ and $\lim _{n \rightarrow \infty} \mathbb{E}_{\mathbb{P}}\left[U\left(g_{n}\right)\right]=\mathbb{E}_{\mathbb{P}}[U(g)]$. In view of the concavity of $U$, one has $\mathbb{E}_{\mathbb{P}}\left[U\left(g_{n}\right)\right] \geq \inf _{k \geq n} \mathbb{E}_{\mathbb{P}}\left[U\left(f_{k}\right)\right]$ for all $n \in$ $\mathbb{N}$. Since $\lim _{n \rightarrow \infty} \inf _{k \geq n} \mathbb{E}_{\mathbb{P}}\left[U\left(f_{k}\right)\right]=\mathbb{E}_{\mathbb{P}}[U(f)]$, we conclude that $\mathbb{E}_{\mathbb{P}}[U(f)] \leq$ $\mathbb{E}_{\mathbb{P}}[U(g)]$. The last inequality combined with the fact that $U$ is strictly increasing contradicts $\mathbb{P}[f>g]=1$. Therefore, we conclude that, $\Pi$-a.s., $f \leq g$.

2.5. Equivalence of (1), (2) and (3) in Theorem 1.3; general case. We shall now tackle the general case $f \in \mathbb{L}_{+}^{0}$, working under the assumption (CONV). Of course $(1) \Rightarrow(2)$ and $(3) \Rightarrow(1)$ are still trivially valid. The proof of $(2) \Rightarrow(3)$ will be reduced to the special case $f=0$, which we have already established, via the following result. 
Lemma 2.4. The following statements are equivalent:

(i) Every sequence of forward-convex combinations of $\left(f_{n}\right)_{n \in \mathbb{N}} \mathbb{L}_{+}^{0}$-converges to $f$.

(ii) Every sequence of forward-convex combinations of $\left(\left|f_{n}-f\right|\right)_{n \in \mathbb{N}} \mathbb{L}_{+}^{0}$ converges to zero.

Proof. As (ii) $\Rightarrow$ (i) is immediate, we only treat the implication (i) $\Rightarrow$ (ii). For $x \in \mathbb{R}$ and $y \in \mathbb{R}$, we denote by $x_{+}$the positive part of $x$ and by $x \wedge y$ the minimum between $x$ and $y$. We shall first argue that every sequence of forward-convex combinations of $\left(\left(f-f_{n}\right)_{+}\right)_{n \in \mathbb{N}} \mathbb{L}_{+}^{0}$-converges to zero. The facts that $0 \leq f_{n} \wedge f \leq f$ for all $n \in \mathbb{N}$ and $\mathbb{L}_{+}^{0}-\lim _{n \rightarrow \infty} f_{n}=f$, coupled with Proposition 2.3 , imply that whenever a sequence of forward-convex combinations of $\left(f_{n} \wedge f\right)_{n \in \mathbb{N}} \mathbb{L}_{+}$-converges, the limit is $f$. Since $\left(f-f_{n}\right)_{+}=f-f \wedge f_{n}$, it follows that whenever a sequence of forward-convex combinations of $\left(\left(f-f_{n}\right)_{+}\right)_{n \in \mathbb{N}} \mathbb{L}_{+}$-converges, the limit is zero. From the special case of Theorem 1.3 that we have established previously, it actually follows that every sequence of forward-convex combinations of $\left(\left(f-f_{n}\right)_{+}\right)_{n \in \mathbb{N}} \mathbb{L}_{+^{-}}$ converges to zero. Note also that this implies that every sequence of forward-convex combinations of $\left(\left(f \wedge f_{n}\right)_{+}\right)_{n \in \mathbb{N}} \mathbb{L}_{+}$-converges to $f$. We now proceed to show that every sequence of forward-convex combinations of $\left(\left(f_{n}-f\right)_{+}\right)_{n \in \mathbb{N}} \mathbb{L}_{+}$-converges to zero, which will complete the argument. The fact that $\left(f_{n}-f\right)_{+}=f_{n}-f \wedge f_{n}$ for all $n \in \mathbb{N}$ and Proposition 2.3 imply that whenever a sequence of forwardconvex combinations of $\left(\left(f_{n}-f\right)_{+}\right)_{n \in \mathbb{N}} \mathbb{L}_{+}$-converges, its limit is zero. Once again, by the special case of Theorem 1.3 that we have established previously, it actually follows that every sequence of forward-convex combinations of $\left(\left(f_{n}-f\right)_{+}\right)_{n \in \mathbb{N}}$ $\mathbb{L}_{+}$-converges to zero. This completes the proof.

In view of the result of Lemma 2.4 and the treatment in subsection 2.3, we obtain the existence of $\mathbb{Q} \in \Pi$ such that $\sup _{n \in \mathbb{N}} \mathbb{E}_{\mathbb{Q}}\left[\left|f_{n}-f\right|\right]<\infty$ and $\lim _{n \rightarrow \infty} \mathbb{E}_{\mathbb{Q}}\left[\left|f_{n}-f\right|\right]=$ 0 . Replacing $\mathbb{Q}$, if necessary, by $\mathbb{Q}^{\prime} \in \Pi$ defined via $\mathrm{d} \mathbb{Q}^{\prime} / \mathrm{d} \mathbb{P}=c(1+f)^{-1}$ where $c=\left(\mathbb{E}_{\mathbb{Q}}\left[(1+f)^{-1}\right]\right)^{-1}$, we may further assume that $\mathbb{E}_{\mathbb{Q}}[f]<\infty$; in other words, $\sup _{n \in \mathbb{N}} \mathbb{E}_{\mathbb{Q}}\left[f_{n}\right]<\infty$ and $\lim _{n \rightarrow \infty} \mathbb{E}_{\mathbb{Q}}\left[\left|f_{n}-f\right|\right]=0$.

2.6. Proof of the claims after the equivalences. To begin with, assume that any one of the equivalent statements of Theorem 1.3 is not valid. Then, we must have that $\{f\} \subsetneq \mathcal{C}$, since $\mathcal{C}=\{f\}$ is actually statement (2). Then, Proposition 2.3 implies that for all $g \in \mathcal{C}$ we have, $\Pi$-a.s., $f \leq g$.

Continuing, assume the validity of any of the equivalent statements of Theorem 1.3. The following result will help to establish all the properties of $\mathcal{C}_{1}$ that are mentioned in Theorem 1.3 .

Lemma 2.5. Let $\mathcal{C}_{1}^{\prime} \subseteq \mathbb{L}_{+}$be the set on the right-hand side of (1.1). If $\mathbb{Q} \in \Pi$ is such that condition $(3)$ of Theorem 1.3 holds, then $\mathcal{C}_{1}^{\prime}$ is $\mathbb{L}_{+}^{1}(\mathbb{Q})$-compact.

Proof. First of all, since $\sup _{n \in \mathbb{N}} \mathbb{E}_{\mathbb{Q}}\left[f_{n}\right]<\infty$, which in particular implies that $\mathbb{E}_{\mathbb{Q}}[f]<\infty$ by Fatou's lemma, it is clear that $\sup _{g \in \mathcal{C}_{1}^{\prime}} \mathbb{E}_{\mathbb{Q}}[g]<\infty$; in particular, $\mathcal{C}_{1}^{\prime} \subseteq \mathbb{L}_{+}^{0}$.

We shall show that any sequence $\left(g_{k}\right)_{k \in \mathbb{N}}$ in $\mathcal{C}_{1}^{\prime}$ has an $\mathbb{L}_{+}^{1}(\mathbb{Q})$-convergent subsequence. For all $k \in \mathbb{N}$, write $g_{k}=\sum_{n \in \mathbb{N}} \alpha_{k, n} f_{n}+\left(1-\sum_{n \in \mathbb{N}} \alpha_{k, n}\right) f$, where $\alpha_{k}=\left(\alpha_{k, n}\right)_{n \in \mathbb{N}} \in \triangle^{\mathbb{N}}$. By a diagonalization argument, we can find a subsequence of $\left(g_{k}\right)_{k \in \mathbb{N}}$, which we shall still denote by $\left(g_{k}\right)_{k \in \mathbb{N}}$, such that $\alpha_{n}:=\lim _{k \rightarrow \infty} \alpha_{k, n}$ 
exists for all $n \in \mathbb{N}$. Fatou's lemma implies that $\alpha=\left(\alpha_{n}\right)_{n \in \mathbb{N}} \in \triangle^{\mathbb{N}}$. Let $g:=$ $\sum_{n \in \mathbb{N}} \alpha_{n} f_{n}+\left(1-\sum_{n \in \mathbb{N}} \alpha_{n}\right) f$. We shall show that $\lim _{k \rightarrow \infty} \mathbb{E}_{\mathbb{Q}}\left[\left|g_{k}-g\right|\right]=0$. For $\epsilon>0$, pick $N=N(\epsilon) \in \mathbb{N}$ such that $\sup _{n \in \mathbb{N}} \mathbb{E}_{\mathbb{Q}}\left[\left|f_{N+n}-f\right|\right] \leq \epsilon / 2$. Define $g^{(N)}:=$ $\sum_{n=1}^{N} \alpha_{n} f_{n}+\left(1-\sum_{n=1}^{N} \alpha_{n}\right) f$, as well as $g_{k}^{(N)}:=\sum_{n=1}^{N} \alpha_{k, n} f_{n}+\left(1-\sum_{n=1}^{N} \alpha_{k, n}\right) f$ for all $k \in \mathbb{N}$. Observe that

$$
\mathbb{E}_{\mathbb{Q}}\left[\left|g^{(N)}-g\right|\right]=\mathbb{E}_{\mathbb{Q}}\left[\left|\sum_{n \in \mathbb{N}} \alpha_{N+n}\left(f_{N+n}-f\right)\right|\right] \leq \sum_{n \in \mathbb{N}} \alpha_{N+n} \mathbb{E}_{\mathbb{Q}}\left[\left|f_{N+n}-f\right|\right] \leq \frac{\epsilon}{2} .
$$

Similarly, $\mathbb{E}_{\mathbb{Q}}\left[\left|g_{k}^{(N)}-g_{k}\right|\right] \leq \epsilon / 2$ holds for all $k \in \mathbb{N}$. Furthermore,

$$
\limsup _{k \rightarrow \infty} \mathbb{E}_{\mathbb{Q}}\left[\left|g_{k}^{(N)}-g^{(N)}\right|\right] \leq \limsup _{k \rightarrow \infty}\left(\sum_{n=1}^{N}\left|\alpha_{k, n}-\alpha_{n}\right| \mathbb{E}_{\mathbb{Q}}\left[\left|f_{n}-f\right|\right]\right)=0 .
$$

It follows that $\lim \sup _{k \rightarrow \infty} \mathbb{E}_{\mathbb{Q}}\left[\left|g_{k}-g\right|\right] \leq \epsilon$. Since $\epsilon>0$ is arbitrary, we have $\lim _{k \rightarrow \infty} \mathbb{E}_{\mathbb{Q}}\left[\left|g_{k}-g\right|\right]=0$.

To finish the proof of Theorem [1.3 it remains to show that $\mathcal{C}_{1}=\mathcal{C}_{1}^{\prime}$ and that the $\mathbb{L}_{+}^{0}$-topology coincides with the $\mathbb{L}_{+}^{1}(\mathbb{Q})$-topology on $\mathcal{C}_{1}$. First of all, since $f \in \mathcal{C}_{1}, f_{n} \in \mathcal{C}_{1}$ for all $n \in \mathbb{N}$, and $\mathcal{C}_{1}$ is closed, we have $\mathcal{C}_{1}^{\prime} \subseteq \mathcal{C}_{1}$. On the other hand, $\operatorname{conv}\left(\left\{f_{1}, f_{2}, \ldots\right\}\right) \subseteq \mathcal{C}_{1}^{\prime}$; since $\mathcal{C}_{1}^{\prime}$ is $\mathbb{L}_{+}^{0}$-closed by Lemma 2.5, $\mathcal{C}_{1}=$ $\overline{\operatorname{conv}}\left(\left\{f_{1}, f_{2}, \ldots\right\}\right) \subseteq \mathcal{C}_{1}^{\prime}$. Therefore, $\mathcal{C}_{1}=\mathcal{C}_{1}^{\prime}$. Finally, let $\left(g_{k}\right)_{k \in \mathbb{N}}$ be a $\mathcal{C}_{1}$-valued and $\mathbb{L}_{+}^{0}$-convergent sequence, and call $g:=\mathbb{L}_{+}^{0}-\lim _{k \rightarrow \infty} g_{k} \in \mathcal{C}_{1}$. Lemma 1.1 implies that every subsequence of $\left(g_{k}\right)_{k \in \mathbb{N}}$ has a further subsequence that is $\mathbb{L}_{+}^{1}(\mathbb{Q})$ convergent. All the latter subsequences have to $\mathbb{L}_{+}^{1}(\mathbb{Q})$-converge to $g$, which means that $\left(g_{k}\right)_{k \in \mathbb{N}} \mathbb{L}_{+}^{1}(\mathbb{Q})$-converges to $g$.

\section{REFERENCES}

1. Klaus Bichteler, Stochastic integrators, Bull. Amer. Math. Soc. (N.S.) 1 (1979), no. 5, 761-765. MR537627 (82k:60122)

2. Stochastic integration and $L^{p}$-theory of semimartingales, Ann. Probab. 9 (1981), no. 1, 49-89. MR606798 (82g:60071)

3. W. Brannath and W. Schachermayer, A bipolar theorem for $\mathbb{L}_{+}^{0}(\Omega, \mathcal{F}, \mathbb{P})$, Séminaire de Probabilités, XXXIII, Lecture Notes in Math., vol. 1709, Springer, Berlin, 1999, pp. 349-354. MR.1768009(2001d:46019)

4. A. V. Buhvalov and G. Ja. Lozanovskiı̌, Sets closed in measure in spaces of measurable functions, Dokl. Akad. Nauk SSSR 212 (1973), 1273-1275. MR0346507 (49:11232)

5. F. Delbaen and W. Schachermayer, A compactness principle for bounded sequences of martingales with applications, Seminar on Stochastic Analysis, Random Fields and Applications (Ascona, 1996), Progr. Probab., vol. 45, Birkhäuser, Basel, 1999, pp. 137-173. MR.1712239 (2001d:60044)

6. Freddy Delbaen and Walter Schachermayer, A general version of the fundamental theorem of asset pricing, Math. Ann. 300 (1994), no. 3, 463-520. MR1304434 (95m:90022b)

7. C. Dellacherie, Un survol de la théorie de l'intégrale stochastique, Stochastic Process. Appl. 10 (1980), no. 2, 115-144. MR.587420 (83i:60069)

8. Damir Filipović, Michael Kupper, and Nicolas Vogelpoth, Separation and duality in locally $L^{0}$-convex modules, J. Funct. Anal. 256 (2009), no. 12, 3996-4029. MR2521918(2011b:46081)

9. N. J. Kalton, N. T. Peck, and James W. Roberts, An F-space sampler, London Mathematical Society Lecture Note Series, vol. 89, Cambridge University Press, Cambridge, 1984. MR808777 (87c:46002)

10. Constantinos Kardaras, Numéraire-invariant preferences in financial modeling, Ann. Appl. Probab. 20 (2010), no. 5, 1697-1728. MR2724418(2011h:60077)

11. J. Komlós, A generalization of a problem of Steinhaus, Acta Math. Acad. Sci. Hungar. 18 (1967), 217-229. MR0210177 (35:1071) 
12. D. Kramkov and W. Schachermayer, The asymptotic elasticity of utility functions and optimal investment in incomplete markets, Ann. Appl. Probab. 9 (1999), no. 3, 904-950. MR.1722287 (2001d:91099)

13. E. M. Nikišin, A certain problem of Banach, Dokl. Akad. Nauk SSSR 196 (1971), 774-775. MR0277950 (43:3683)

14. C. Stricker, Une caractérisation des quasimartingales, Séminaire de Probabilités, IX (Seconde Partie, Univ. Strasbourg, Strasbourg, années universitaires 1973/1974 et 1974/1975), Lecture Notes in Math., Vol. 465, Springer, Berlin, 1975, pp. 420-424. MR0423516 (54:11492)

15. Gordan Žitković, Convex compactness and its applications, Mathematics and Financial Economics 3 (2010), no. 1, 1-12. MR2651515

Department of Mathematics and Statistics, Boston University, 111 Cummington Street, Boston, Massachusetts 02215

E-mail address: kardaras@bu.edu

Department of Mathematics, University of Texas at Austin, 1 University Station, C1200, Austin, Texas 78712

E-mail address: gordanz@math.utexas.edu 